\title{
Interstitial Pulmonary Amyloidosis with Waldenström's Macroglobulinemia
}

\author{
Kayoko Okamura ${ }^{1}$, Junji Takiguchi ${ }^{1}$, Hirokazu Sakamoto ${ }^{1}$ and Nobutaka Inoue ${ }^{2}$ \\ Key words: pulmonary amyloidosis, Waldenström's macroglobulinemia
}

(Intern Med 54: 353-354, 2015)

(DOI: 10.2169/internalmedicine.54.2905)

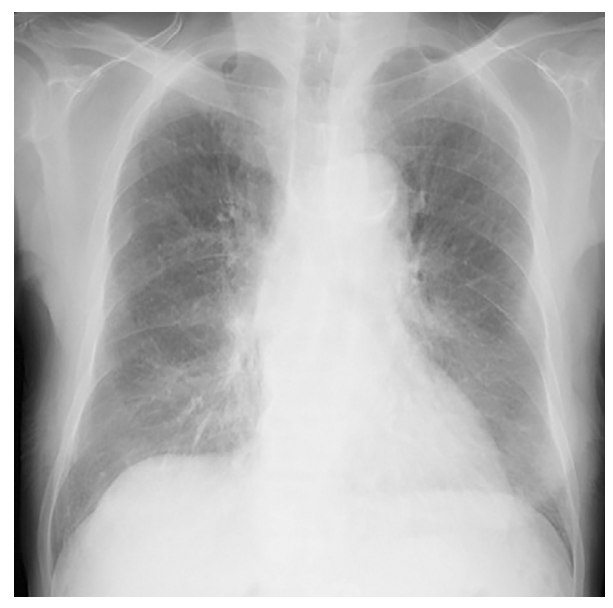

Picture 1.
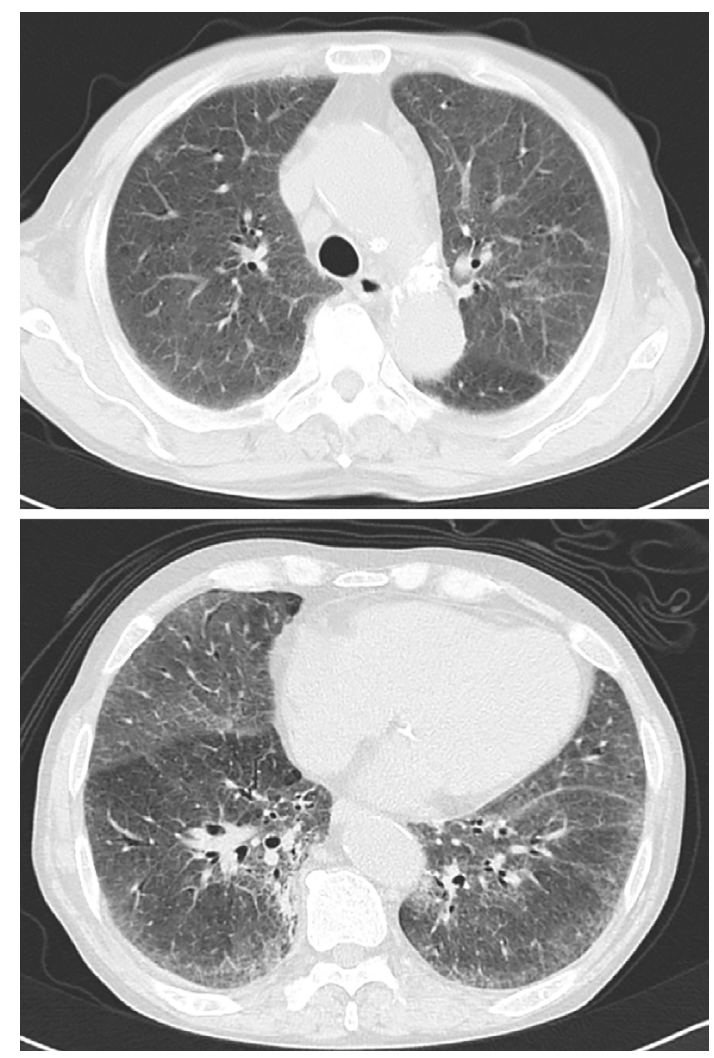

Picture 2.
A 65-year-old man presented with progressive dyspnea. A chest X-ray showed bilateral interstitial shadows (Picture 1), and computed tomography demonstrated prominent thickening of the interlobular septum and ground-glass opacity in the lungs (Picture 2). In addition, the serum IgM level was extremely high $(3,060 \mathrm{mg} / \mathrm{dL})$, and immunoelectrophoresis showed the presence of IgM lambda in the serum. Meanwhile, bone marrow aspirate contained $36 \%$ small lymphocytes, and immunohistochemistry using transbronchial lung biopsy specimens confirmed the deposition of amyloid and
$\operatorname{IgM} \lambda$ type within the alveolar septa (Picture 3). Ultimately, the patient was diagnosed with Waldenström's macroglobulinemia associated with pulmonary diffuse alveolar septal amyloidosis. It has been reported that only $2.2 \%$ of patients with a high monoclonal IgM titer present with amyloidosis with $10 \%$ of such cases of amyloidosis involving in the lungs (1). Diffuse deposition of amyloid in the alveolar septum is rare in patients with pulmonary amyloidosis, although multiple small nodules are frequently observed (2). The present case highlights the substantial variability in the 


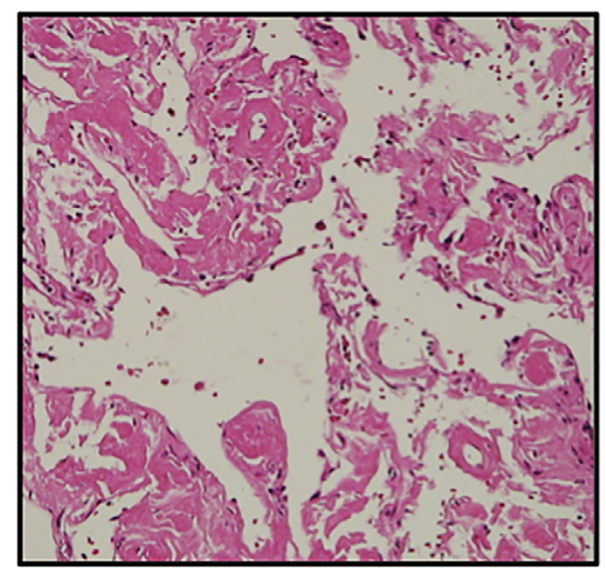

Hematoxylin-Eosin

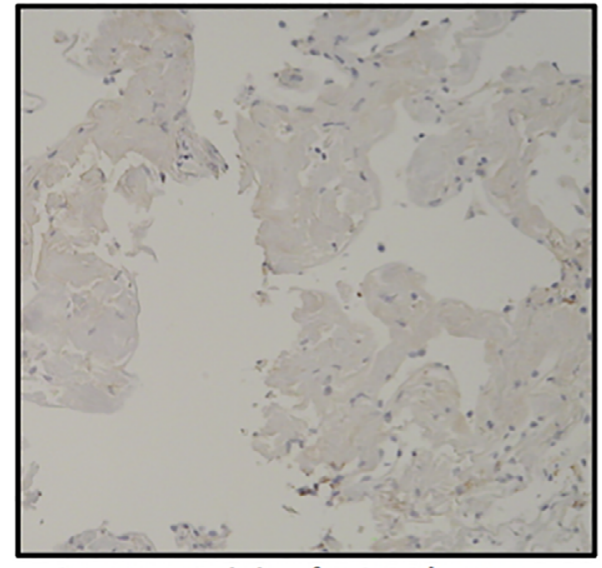

Immunostaining for IgM kappa

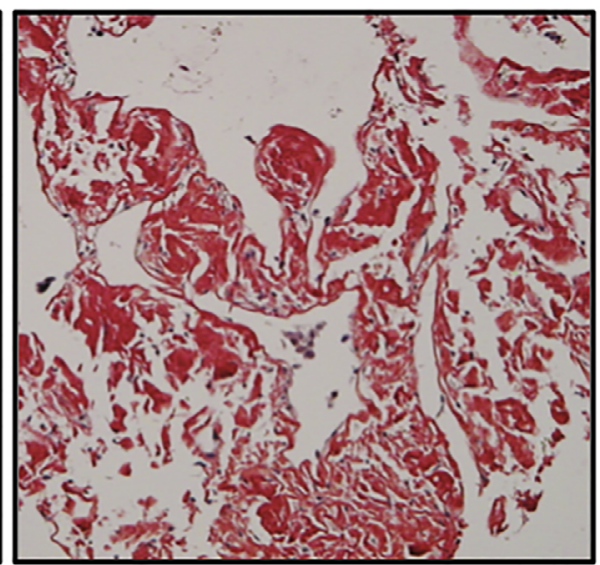

Direct Fast Scarlet staining for amyloid

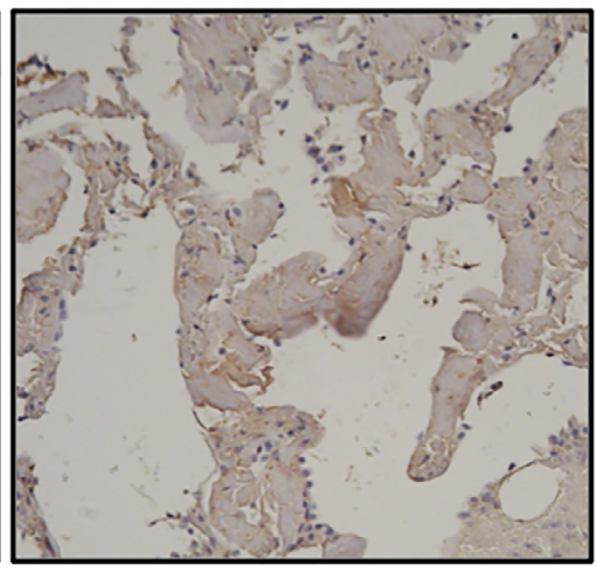

Immunostaining for $\lg M \lambda$

Picture 3.

clinical features of this disease.

The authors state that they have no Conflict of Interest (COI).

\section{References}

1. Gertz MA, Kyle RA, Noel P. Primary systemic amyloidosis: a rare complication of immunoglobulin $\mathrm{M}$ monoclonal gammopathies and Waldenström's macroglobulinemia. J Clin Oncol 11: 914-920, 1993.

2. Pickford HA, Swense SJ, Utz JP. Thoracic cross-sectional imaging of amyloidosis. Am J Roentgenol 168: 351-355, 1997.

(C) 2015 The Japanese Society of Internal Medicine http://www.naika.or.jp/imonline/index.html 